\title{
一种新颖的红外与可见光图像融合方法
}

\author{
李婵飞 ${ }^{1}$, 刘文晶 ${ }^{2}$ \\ (1. 汉口学院, 湖北 武汉 430212; 2. 重庆邮电大学 移通学院, 重庆 401520)
}

\begin{abstract}
摘要: 为了提升红外和可见光图像融合效果, 提出了一种新颖的融合方法。对可见光图像利用支持 向量机和腐蚀澎胀算法在图像分块的基础上进行分割获取含有重要场景信息的特征子图像和灰度场 景子图像; 对红外图像进行热目标边缘提取并增强, 结合前述特征子图像和最大类间方差法获取边 缘增强的目标子图像, 特征子图像和灰度场景子图像。利用小波包分别对两特征子图像, 两场景子 图像进行融合。融合过程中, 根据子图像特点择取不同融合准则, 并对高频融合系数进行系数修正 使其更为准确可靠。将红外热目标注入到前述融合结果中获取最终融合图像。实验结果表明, 提出 算法从主、客观评价上都要优于对比算法。
\end{abstract}

关键词: 支持向量机; 腐蚀膨胀; 小波包; 图像融合; 边缘增强

中图分类号：TN911.73 文献标识码：A 文章编号：1001-8891(2020)04-0370-08

\section{Novel Fusion Method for Infrared and Visible Light Images}

\author{
LI Chanfei ${ }^{1}$, LIU Wenjing ${ }^{2}$ \\ (1. Hankou University, Wuhan 430212, China;
}

2. College of Mobile Telecommunications, Chongqing University of Posts and Telecommunications, Chongqing 401520, China)

\begin{abstract}
To improve the fusion effect of infrared and visible light images, a novel fusion method is proposed. The visible light image is segmented into feature subimages with important scene information and grayscale scene subimages using a support vector machine and corrosion expansion algorithm based on the image block. The hot target edge of the infrared image is extracted and enhanced. Target, feature, and gray background subimages are obtained by combining the information of the former feature subimage with the maximum interclass variance method. Two feature subimages(grayscale scene and gray background) are fused by wavelet packet transform. During fusion, different fusion rules are implemented according to the characteristics of the subimages. Additionally, high frequency fusion coefficients are modified to render them more accurate and reliable. The infrared thermal target is injected into the previous fusion result to obtain the final fusion image. Experimental results show that the proposed algorithm is superior to other algorithms both in subjective and objective evaluations.
\end{abstract}

Key words: support vector machine, corrosion expansion, wavelet packet transform, image fusion, edge enhancement

\section{0 引言}

多传感器图像融合是指对同一场景的多幅图像 信息进行融合生成单幅图像的过程, 使得融合后的图 像更加符合人类对视觉的感知。近些年来, 图像融合 被广泛应用于生物识别、无损检测、军事监视、临床 医学、遥感信息等领域 ${ }^{[1-7]}$ 。
红外和可见光图像融合是多传感器图像融合研 究的重要组成部分。红外图像主要是利用红外传感器 来记录不同物体的热辐射信息, 能较好地探测隐蔽的 热目标, 受环境变化影响较小。可见光图像是用来记 录物体光谱信息的反射特性, 主要包含的是图像的场 景细节和边缘信息 ${ }^{[8]}$ 。将红外与可见光图像进行融合, 能够有效地将红外图像中的目标信息和可见光图像 
中的目标场景、细节边缘信息相结合，增强对场景的 理解, 突出热目标。

根据应用场合的不同, 红外与可见光图像融合可 以分为 3 个层次: 像素级, 特征级和符号级 ${ }^{[9]}$ 。在红 外与可见光图像融合过程中, 基于像素级的多分辨率 分析融合是一类应用广泛的融合方法。文献[10-11]将 金字塔变换（Laplacian pyramid transform, LPT）应用 于图像融合, 但是 LPT 会产生大量的咒余信息, 对图 像的方向特性不能有效表达; 文献[12]将小波变换 (discrete wavelet transform, DWT) 应用于图像融合, 小波变换可以克服 LPT 的缺点, 但是捕捉到图像方向 信息有限 (仅水平, 垂直和对角方向), 且会在图像 局部区域产生振铃效应; 文献[13]将平稳小波变换 （stationary wavelet transform, SWT）应用于图像融合, SWT 能克服 DWT 产生的振铃效应问题, 但其分解策 略和小波一样, 仅对每层的低频成分重新分解, 图像 时频分辨率不高; 文献[14]将轮廓波变换 (contourlet transform, CT）应用于图像融合, 但是由于缺乏平移 不变性, 导致 Gibbs 效应的产生; 文献[15]将非下采 样轮廓波变换 (nonsubsampled contourlet transform, NSCT）应用于图像融合, NSCT 能克服 CT 的缺陷, 但是计算效率偏低, 比较耗时。文献[16-17]将多分辨 率分析工具和图像分割结合应用于图像融合, 尽管该 类算法融合图像可以较好地保持红外目标信息, 但是 对于红外图像的重要特征信息和可见光图像中特定 的重要场景细节信息不能有效保持。因此, 将红外图 像的重要特征信息和可见光图像中特定的场景细节 信息从源图像中提取出来引入图像融合过程是非常 必要的。

鉴于上述考虑, 提出了一种新颖的红外与可见光 图像融合方法。利用支持向量机 (support vector machine, SVM $)^{[18]}$ 和图像腐蚀膨胀算法在图像分块的 基础上将可见光图像分割为场景细节特征丰富的区 域特征子图像和灰度场景子图像, 在 Canny 检测算子 和最大内间方差方法基础上结合可见光特征子图像 信息引入 Laplacian 图像锐化算法将红外图像分割为 边缘增强的目标子图像, 特征子图像和灰度场景子图 像。然后分别将两特征子图像, 两场景子图像利用小 波包 ${ }^{[19]}$ 分解并择取不同的融合规则融合获得融合背 景图像, 将红外目标子图像融入到融合背景图像中获 取最终融合图像。

\section{1 小波包变换原理}

多分辨率分析可以对信号进行有效的时频分解, 但是其每层的分解都是在低频成分进行, 因此它对高
频段的成分分辨率影响较低。小波包分解 (wavelet packet）是在多分辨率分析基础上发展起来的一种精 细的信号分析方法, 它能够为信号提供一种更为精 细的分解方法, 不仅对信号进行多频带划分, 而且 对多分辨率分析没有分解的高频成分进行再分解, 提高了信号的时频分辨率, 是一种更有价值的信号 分析工具。

小波包变换主要分为分解和重构两个步骤, 其分 解和重构公式分别如(1)、(2)所示:

$$
\begin{gathered}
\left\{\begin{array}{l}
d_{k}^{j+1,2 n}=\sum_{l} h_{0(2 l-k)} d_{l}^{j, n} \\
d_{k}^{j+1,2 n+1}=\sum_{l} h_{1(2 l-k)} d_{l}^{j, n}
\end{array}\right. \\
d_{l}^{j, n}=\sum_{k}\left[h_{0(2 l-k)} d_{k}^{j+1,2 n}+h_{1(2 l-k)} d_{k}^{j+1,2 n+1}\right] \\
=\sum_{k}\left[g_{0(2 l-k)} d_{k}^{j+1,2 n}+g_{1(2 l-k)} d_{k}^{j+1,2 n+1}\right]
\end{gathered}
$$

式中: $h_{0}(2 l-k)$ 和 $h_{1}(2 l-k)$ 是多分辨率分析滤波器系 数; $g_{0}(2 l-k)$ 和 $g_{1}(2 l-k)$ 分别为小波包重构过程中的 低通和高通滤波器组。

\section{2 新颖的红外与可见光图像融合方法}

一种新颖的红外与可见光图像融合方法主要利 用支持向量机和图像腐蚀膨胀算法在图像分块的基 础上将可见光图像分割为具有重要特征场景的特征 子图像和灰度场景子图像, 在 Canny 检测算子和最大 内间方差方法基础上结合 Laplacian 算子图像锐化算 法和可见光特征子图像将红外图像分割为边缘增强 的目标子图像, 特征子图像和灰度场景子图像。然后 分别将两特征子图像, 两场景子图像利用小波包分解 并择取不同的融合规则融合获得融合背景图像, 将红 外目标子图像融入到融合背景图像中获取最终融合 图像。该算法流程图如图 1 所示。

\section{1 可见光图像分割}

在可见光图像中, 有价值的除了场景边缘细节和 光谱信息之外, 还应包含一些特定的重要场景特征信 息。在红外和可见光图像融合过程中, 如果将可见光 图像作为一个整体和红外图像融合难以有效表达其 特定场景特征信息, 因此有必要根据可见光图像特点 和图像融合目的对其进行分割。

由图 2(a)可见光源图像可知, 烟图和屋顶等特征 信息应该是图像融合过程中感兴趣的重要场景特征 信息, 如果利用简单的像素级融合不能有效地保持其 丰富的场景细节边缘特征。因此提出利用 SVM 和图 像腐蚀膨胀算法在图像分块的基础上对可见光图像 进行特征子图像的划分。首先利用图像分块获取含有 
烟冲、屋顶的子图像块, 再利用支持向量机将该子图 像像素点进行分类以剔除烟图、屋顶之外的场景信 息, 从而得到图像(b)。由图(b)可知, 该子图像虽然包 含烟图、屋顶信息, 还包含一些其他的小零星场景点。
为了得到尽可能准确的烟图、屋顶子特征图像, 在(b) 的基础上结合图像分块(c) ((c)为(b)图像分块之后的 图像）和腐蚀膨胀算法可以得到比较准确的含有烟 图、屋顶的特征子图像(d)。

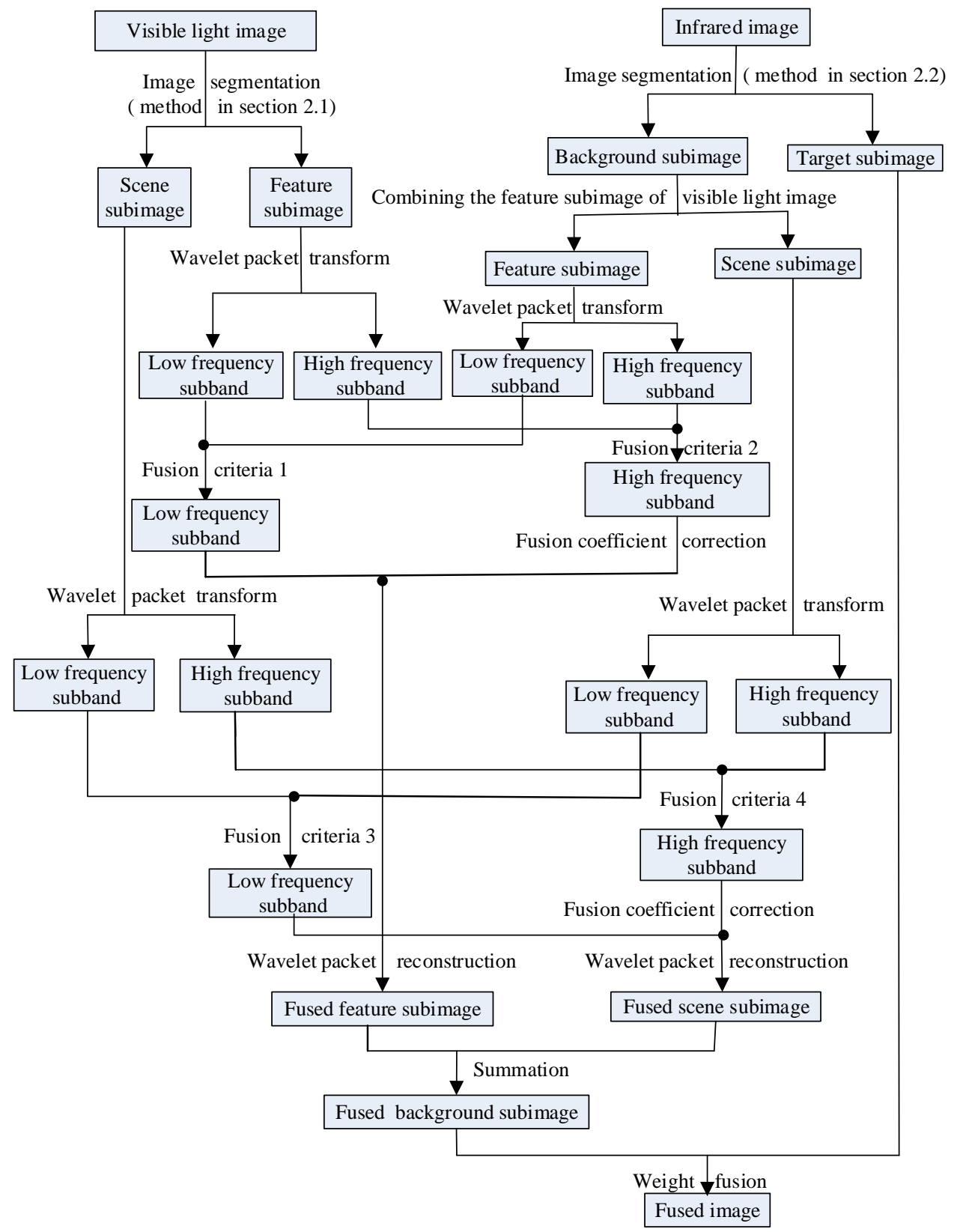

图 1 图像融合算法流程图
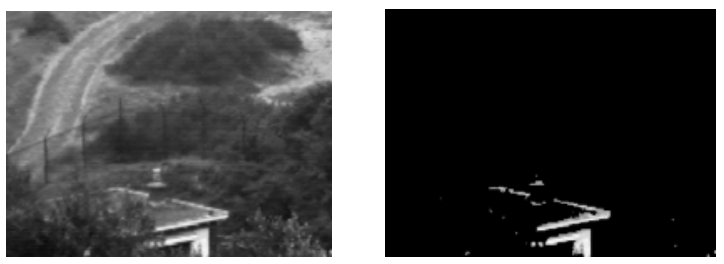

Fig.1 Image fusion method flow

(b) 支持向量机分类之后子图像

(c) 分块之后子图像

(d) 腐蚀膨胀之后特征子图像

(a) Original visible light image (b) Subimage of SVM classification (c) Subimage of blocking (d) Feature subimage of corrosion and expansion 图 2 可见光源图像及其分割图像 Fig.2 Original visible light image and segmentation images 


\section{2 红外目标提取增强}

隐藏的红外热目标是红外图像中最为重要的信 息, 为了在红外和可见光图像融合过程中较好地保持 该热目标信息, 文献[17]提出了一种 Canny 边缘检测 和最大类间方差的目标分割算法。在此基础上, 提出 了一种红外目标提取增强法, 首先利用 Canny 检测算 子获取目标边缘轮廓, 利用 Laplacian 图像锐化算子 对该目标轮廓进行增强, 并计算边缘内部区域均值 Mean; 然后利用最大内间方差法获取源红外图像分割 阈值 $T^{*}$; 如果 Mean $>T^{*}$, 则对应区域为目标图像, 否则为红外背景子图像; 最后根据节 2.1 中的可见光 特征子图像信息将红外背景子图像分割为红外特征 子图像和灰度场景子图像。经过前述步骤得到增强的 红外目标图像, 特征子图像和灰度场景子图像。由图 3 可知, 经过边缘增强的目标人物比原始目标图像更 为清晰明亮, 边缘轮廓更清晰突出。

\section{3 低频融合准则}

图像经小波包分解之后, 低频部分主要包含的是 源图像的概貌纹理近似信息, 体现的是图像的大尺度 特征, 因此低频融合准则的好坏对图像融合质量起着 重要作用。在图 1 中, 根据特征子图像特点, 融合准 则 1 择取系数取大法, 该融合准则计算简单且可以 较好地保持可见光子图像中丰富的烟图、屋顶等重要 场景细节信息。对于场景子图像融合来说, 它们都可 以看作一般的背景区域, 为了更好地保持红外图像的 近似纹理轮廓信息和可见光图像丰富的光谱细节信 息, 其低频子带融合准则 3 择取能量梯度相结合的融 合准则。假设 $E$ 和 $\mathrm{AV}$ 分别表示区域能量和平均梯度, 则其具体融合准则如公式(3)所示:

$$
C(i, j)=\left\{\begin{array}{l}
C_{1}(i, j) \quad E_{1}(i, j) \geq E_{2}(i, j) \\
\begin{cases}C_{1}(i, j) & \mathrm{AV}_{1}(i, j) \geq \operatorname{AV}_{2}(i, j) \\
C_{2}(i, j) & \mathrm{AV}_{1}(i, j)<\operatorname{AV}_{2}(i, j)\end{cases}
\end{array}\right.
$$

\section{4 高频融合准则}

图像经过小波包分解之后, 高频部分主要包含的 是图像的边缘轮廓细节信息, 体现的是图像的小尺度 特征, 且其数据量较大。为了更好地提取图像的细节 信息, 融合准则 2 和 4 均是模值择大法。但是为了使 得融合系数的选取更为准确可靠, 在进行模值择大法 之后, 对融合系数进行系数修正。具体修正方法: 如 果某像素点融合系数来源于红外图像系数, 但其 $3 \times 3$ 邻域内像素点融合系数均来自于可见光图像系数, 则 该像素点融合系数取自于可见光图像融合系数更为合
理, 因此有必要对其进行系数修正; 反之, 做相反的 处理。经过系数修正的融合高频系数更为精确连贯。

\section{3 实验结果与性能评价}

为了验证本文提出方法的有效性, 选取荷兰 TNO Human Factors Research Institute 在不同时刻拍摄的 “UN Camp” 红外和可见光序列图利用 Matlab 软件进 行实验验证。对比方法为: 小波变换融合算法（方法 1 ), 平稳小波变换融合算法 (方法 2), 非下采样轮廓 波融合算法（方法 3), 基本小波包变换融合算法（方 法 4), 文献[17]融合算法（方法 5), 文献[16]融合方 法 (方法 6), 文献[20]融合方法 (方法 7), 提出方法。 其中方法 1 方法 5 的低频融合准则均是均值法, 高 频融合准则是模值取大法。除了方法 3 和方法 7 是自 适应分解层之外，其他方法均采用相同小波基函数 bior4.4 进行 4 层分解。

文章主要通过主观和客观两方面对不同方法融合 性能进行对比评价, 主观直接通过人眼观察对比各方 法融合图像的视觉效果，客观上采用标准差、信息熵、 互信息、空间频率 ${ }^{[21-22]} 4$ 种指标进行评价，同时对不同 方法的运行时间进行对比分析。各方法融合结果如图 4 图 7 所示, 客观评价指标如表 1 和表 2 所示。

观察图 4、5、6、7 可知, 方法 1 方法 4 能综合 可见光和红外图像信息，对红外图像目标人物和可见 光图像屋顶、烟囱等信息有一定程度体现，但其融合 图像亮度不高; 方法 5、6、7 所得融合图像整体效果 相对于前述 4 种方法有较大程度提升，但是在融合图 像中，可见光图像的屋顶、烟图等重要特征信息存在 一定程度的昏暗模糊现象。对比而言, 本文方法的融 合图像整体对比度更高, 反差更大, 红外图像热目标 及其边缘更为清晰明亮突出, 可见光图像的烟图、屋 顶等重要特征信息得到了最大程度的清晰体现, 且较 好地保持了可见光图像的背景光谱信息。因此，本文 融合方法在主观评价上融合效果最好。

比较表 1 、表 2 中不同客观评价指标可知，本文算 法的标准差、信息熵、互信息、空间频率不仅是所有 融合方法中的最大值，且相对于其他融合算法都有较 大幅度提高。由此可知, 本文方法融合图像有最高的 对比度, 包含更为丰富的图像信息且从源图像获取的 信息最多，而且融合图像包含更为丰富的纹理细节特 征，在空间域上最为活跃。另外，在运行时间方面本 文方法的运行时间适中, 满足实时性要求, 从客观上 评价上最为有效。 


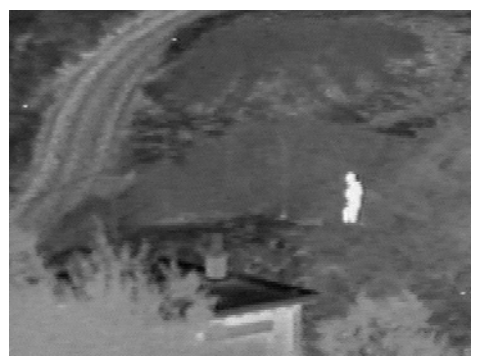

(a) 原始红外图像

(a) Original infrared image

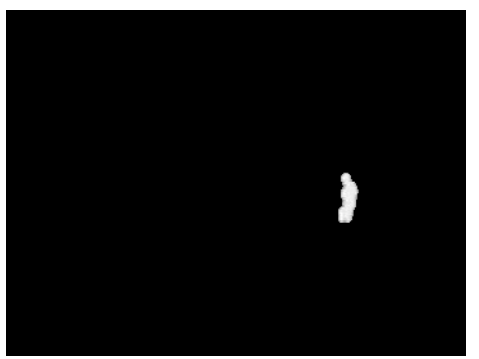

(b) 目标图像

(b) Target image

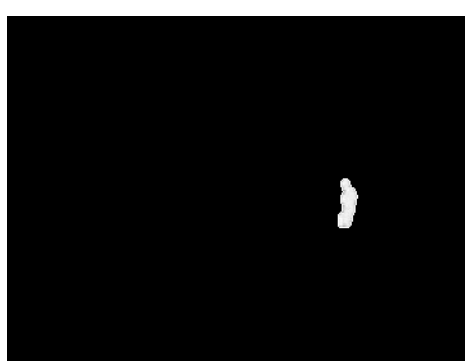

(c) 边缘增强的目标图像

图 3 红外源图像及其目标图像 Fig.3 Original infrared image and target images

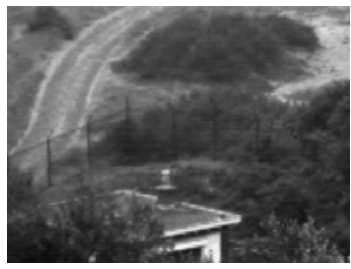

(a) 可见光图像

(a) Original visible light image

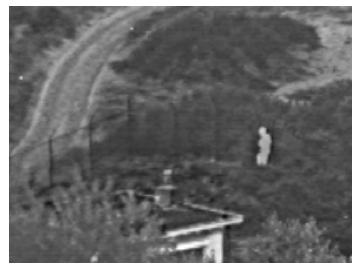

(e) 方法 3 融合图像

(e) Fusion result of method 3

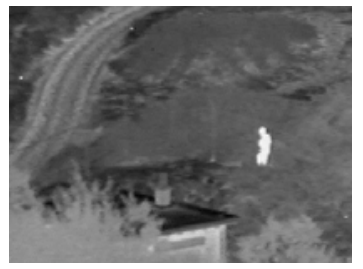

(b) 红外图像

(b) Original infrared image

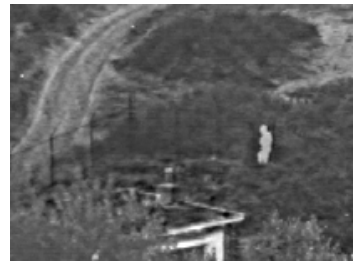

(f) 方法 4 融合图像

(f) Fusion result of method 4

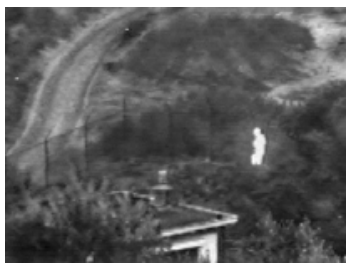

(i) 方法 7 融合图像

(i) Fusion result of method 7

图 4 源图像和不同方法融合图像（1814）

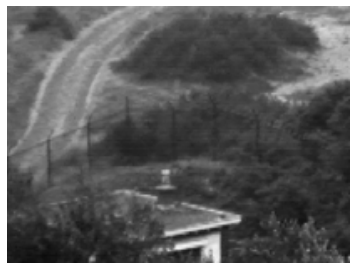

(a) 可见光图像

(a) Original visible light image

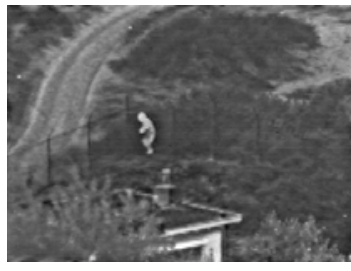

(e) 方法 3 融合图像

(e) Fusion result of method 3

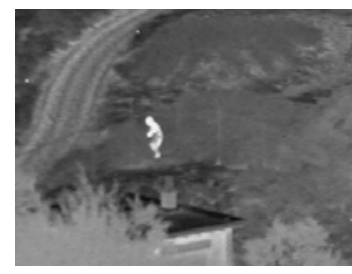

(b) 红外图像

(b) Original infrared image

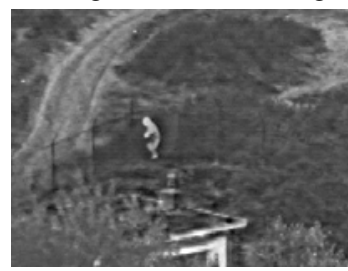

(f) 方法 4 融合图像

(f) Fusion result of method 4

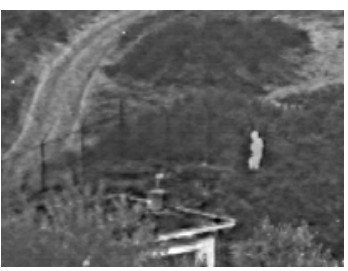

(c) 方法 1 融合图像

(c) Fusion result of method 1

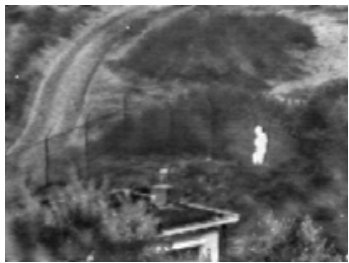

(g) 方法 5 融合图像

(g) Fusion result of method 5

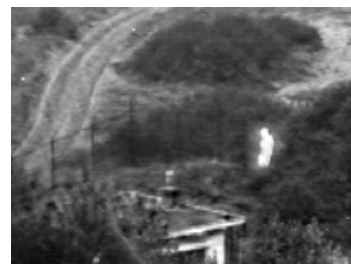

（j）提出方法融合图像

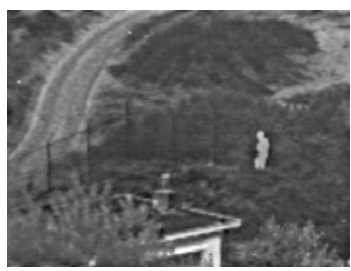

(d) 方法 2 融合图像 (d) Fusion result of method 2

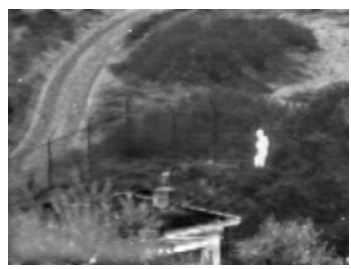

(h) 方法 6 融合图像

(h) Fusion result of method 6

\section{(j) Fusion result of proposed method}

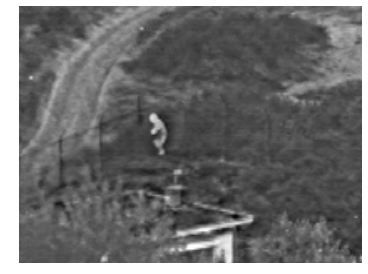

(c) 方法 1 融合图像

(c) Fusion result of method 1

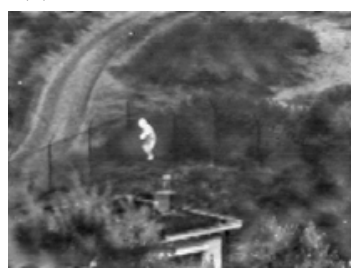

(g) 方法 5 融合图像

(g) Fusion result of method 5

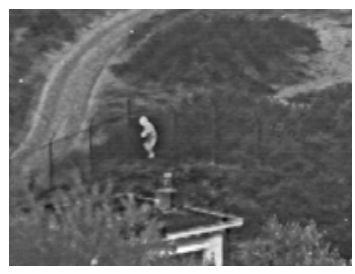

(d) 方法 2 融合图像

(d) Fusion result of method 2

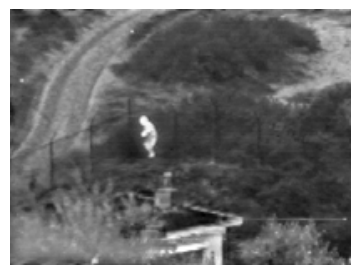

(h) 方法 6 融合图像 (h) Fusion result of method 6 


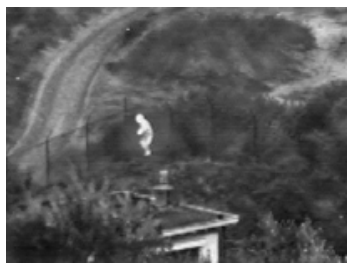

(i) 方法 7 融合图像

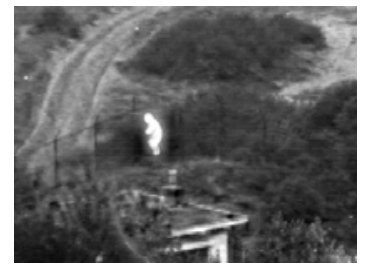

（j）提出方法融合图像
(i) Fusion result of method 7
(j) Fusion result of proposed method

图 5 源图像和不同方法融合图像（1830） Fig.5 Original images and fusion result of different methods（1830）

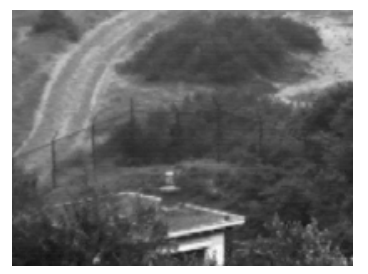

(a) 可见光图像

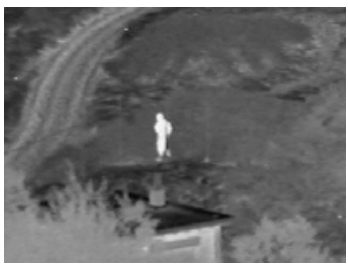

(b) 红外图像

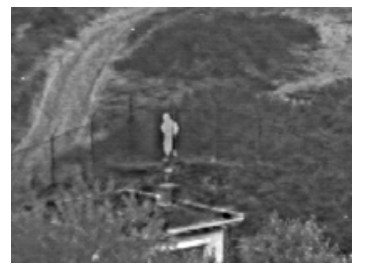

(b) 方法 1 融合图像

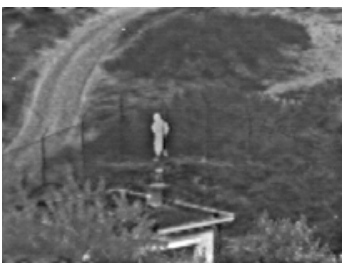

(d) 方法 2 融合图像

(a) Original visible light image (b) Original infrared image
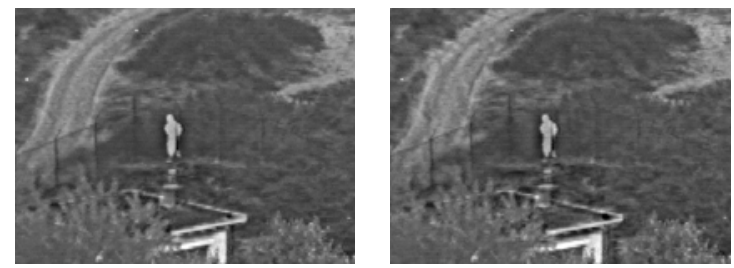

(e) 方法 3 融合图像

(f) 方法 4 融合图像 (c) Fusion result of method 1

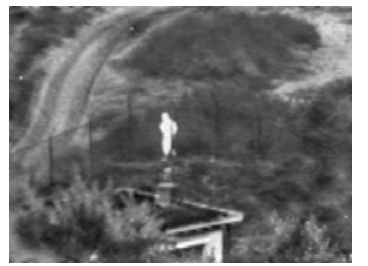

(g) 方法 5 融合图像 (d) Fusion result of method 2

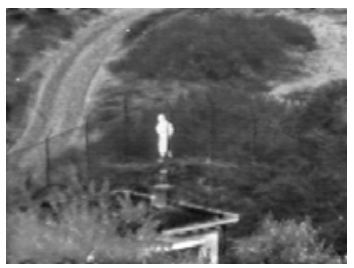

(h) 方法 6 融合图像

(e) Fusion result of method 3 (f) Fusion result of method 4

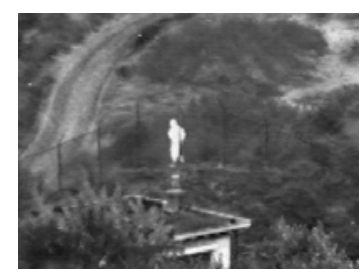

(i) 方法 7 融合图像

(g) Fusion result of method 5 (h) Fusion result of method 6

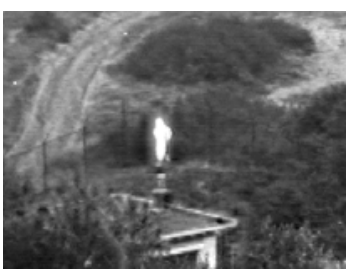

(j) 提出方法融合图像

(i) Fusion result of method 7

图 6 源图像和不同方法融合图像（1819） Fig.6 Original images and fusion result of different methods (1819)

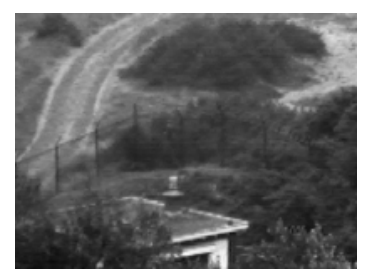

(a) 可见光图像

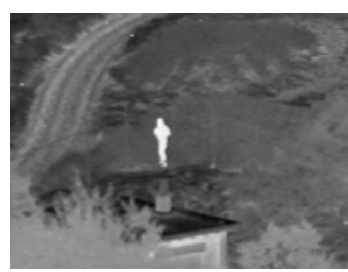

(b) 红外图像

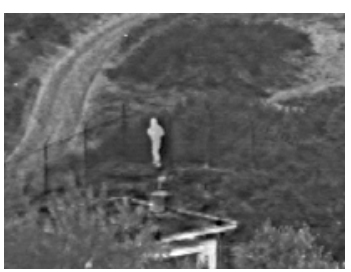

(c) 方法 1 融合图像

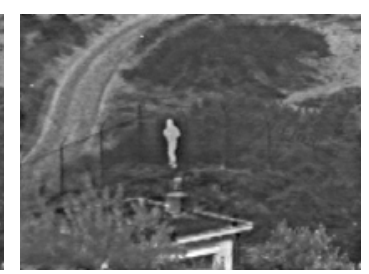

(d) 方法 2 融合图像

(a) Original visible light image (b) Original infrared image (c) Fusion result of method 1 (d) Fusion result of method 2

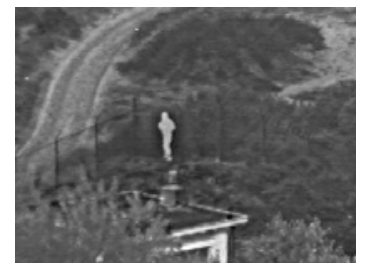

(e) 方法 3 融合图像

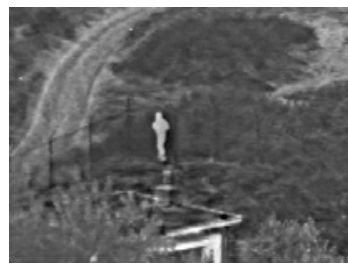

(f) 方法 4 融合图像

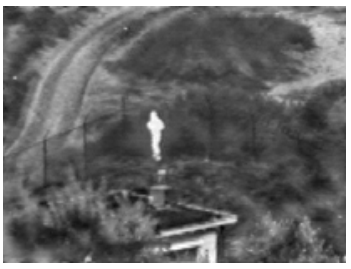

(g) 方法 5 融合图像

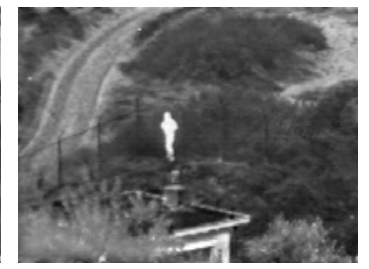

(h) 方法 6 融合图像

(e) Fusion result of method 3 (f) Fusion result of method 4 (g) Fusion result of method 5 (h) Fusion result of method 6 


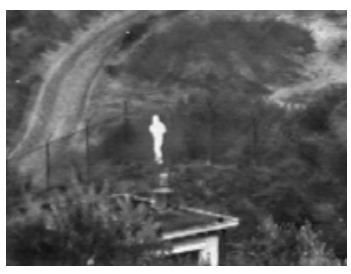

(i) 方法 7 融合图像

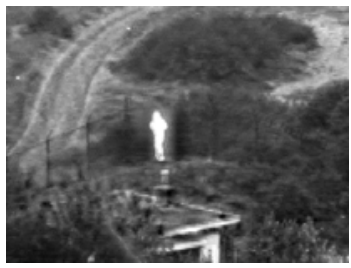

(j) 提出方法融合图像

(i) Fusion result of method 7

(j) Fusion result of proposed method

图 7 源图像和不同方法融合图像（1824） Fig.7 Original images and fusion result of different methods（1824）

表 1 不同方法融合结果客观评价指标 Table 1 Original images and evaluation result of different methods

\begin{tabular}{|c|c|c|c|c|c|c|c|c|c|c|}
\hline \multirow[b]{2}{*}{$\begin{array}{l}\text { Fusion } \\
\text { method }\end{array}$} & \multicolumn{5}{|c|}{ Original images 1814} & \multicolumn{5}{|c|}{ Original images 1830} \\
\hline & Variance & Entropy & $\begin{array}{c}\text { Mutual } \\
\text { information }\end{array}$ & $\begin{array}{c}\text { Spatial } \\
\text { frequency }\end{array}$ & Time/s & Variance & Entropy & $\begin{array}{c}\text { Mutual } \\
\text { information }\end{array}$ & $\begin{array}{c}\text { Spatial } \\
\text { frequency }\end{array}$ & Time/s \\
\hline Method 1 & 28.4961 & 6.6358 & 1.4016 & 13.6033 & 1.4184 & 27.4942 & 6.5822 & 1.3933 & 13.2265 & 1.4979 \\
\hline Method 2 & 28.1855 & 6.6027 & 1.4130 & 11.1875 & 3.0822 & 27.3354 & 6.5576 & 1.3966 & 11.0409 & 3.2400 \\
\hline Method 3 & 27.6539 & 6.5798 & 1.4658 & 12.8191 & 289.0161 & 26.8354 & 6.5336 & 1.4585 & 12.5106 & 286.0095 \\
\hline Method 4 & 28.5103 & 6.6394 & 1.3886 & 13.5414 & 4.8464 & 27.5086 & 6.5864 & 1.3715 & 13.1759 & 4.8647 \\
\hline Method 5 & 36.4245 & 7.0708 & 1.6144 & 11.4964 & 15.2090 & 34.9024 & 7.0183 & 1.5557 & 11.3629 & 14.7684 \\
\hline Method 6 & 38.7058 & 7.1275 & 1.9228 & 11.3512 & 4.45773 & 37.7087 & 7.0867 & 1.9728 & 11.2713 & 4.5622 \\
\hline Method 7 & 34.7133 & 6.9944 & 1.9216 & 12.4670 & 769.8757 & 33.7422 & 6.9721 & 1.9373 & 12.1627 & 762.7120 \\
\hline $\begin{array}{l}\text { Proposed } \\
\text { method }\end{array}$ & 41.9278 & 7.2564 & 2.4999 & 13.5737 & 12.1641 & 40.6025 & 7.1905 & 2.4478 & 13.2752 & 12.8558 \\
\hline
\end{tabular}

表 2 不同方法融合结果客观评价指标 Table 2 Original images and evaluation result of different methods

\begin{tabular}{|c|c|c|c|c|c|c|c|c|c|c|}
\hline \multirow{2}{*}{$\begin{array}{l}\text { Fusion } \\
\text { method }\end{array}$} & \multicolumn{5}{|c|}{ Original images 1819} & \multicolumn{5}{|c|}{ Original images 1824} \\
\hline & Variance & Entropy & $\begin{array}{c}\text { Mutual } \\
\text { information }\end{array}$ & $\begin{array}{c}\text { Spatial } \\
\text { frequency }\end{array}$ & Time/s & Variance & Entropy & $\begin{array}{c}\text { Mutual } \\
\text { information }\end{array}$ & $\begin{array}{c}\text { Spatial } \\
\text { frequency }\end{array}$ & Time/s \\
\hline Method 1 & 27.4579 & 6.5916 & 1.4040 & 13.3174 & 1.4494 & 28.3801 & 6.6339 & 1.4100 & 13.4800 & 1.4215 \\
\hline Method 2 & 27.4682 & 6.5795 & 1.4186 & 11.0705 & 3.1929 & 28.0915 & 6.6051 & 1.4120 & 11.1929 & 3.0029 \\
\hline Method 3 & 26.9848 & 6.5532 & 1.4740 & 12.6448 & 288.5730 & 27.5755 & 6.5807 & 1.4732 & 12.7180 & 286.2214 \\
\hline Method 4 & 27.4843 & 6.5916 & 1.3938 & 13.2938 & 5.0777 & 28.3802 & 6.6371 & 1.3912 & 13.4103 & 4.9163 \\
\hline Method 5 & 35.4093 & 6.9949 & 1.6272 & 11.3896 & 14.7301 & 35.8746 & 7.0289 & 1.5872 & 11.5071 & 13.921413 \\
\hline Method 6 & 36.8929 & 7.0666 & 1.9428 & 11.2554 & 4.5397 & 38.3376 & 7.1085 & 1.9329 & 11.2750 & 4.3534 \\
\hline Method 7 & 33.0098 & 6.8833 & 1.8384 & 12.3023 & 770.0615 & 33.9537 & 6.9448 & 1.8802 & 12.3775 & 765.4237 \\
\hline $\begin{array}{l}\text { Proposed } \\
\text { method }\end{array}$ & 40.6409 & 7.1877 & 2.5030 & 13.3916 & 12.7770 & 41.4259 & 7.2179 & 2.4674 & 13.5591 & 12.9840 \\
\hline
\end{tabular}

综合主、客观评价可知, 本文方法是对比方法中 性能最优的方法。

\section{4 结论}

为了提升红外与可见光图像融合效果, 在融合图 像中更好地突出红外热目标信息, 更高效地体现可见 光图像重要的场景特征细节信息并最大程度地保持 可见光图像丰富的光谱信息, 提出了一种新颖的图像 融合算法。对于红外图像, 不仅提取了红外热目标, 且对该目标边缘利用 Laplacian 图像锐化算法进行边 376
缘增强; 对于可见光图像, 结合支持向量机和腐蚀膨 胀算法在图像分块的基础上提取包含重要场景信息 特征子图像。结合小波包变换对不同的红外、可见光 子图像运用不同的融合规则融合, 并对高频融合系数 进行系数修正使其更为精确可靠。实验结果表明, 本 文的融合方法无论是从主观视觉效果还是客观评价 指标上都要优于对比方法。

\section{参考文献:}

[1] Phamila Y A V, Amutha R. Discrete cosine transform based fusion of multifocus images for visual sensor networks[J]. Signal Process, 2014, 95: 161-170. 
[2] Gangapure V N, Banerjee S, Chowdhury A S. Steerable local frequency based multispectral multifocus image fusion[J]. Inf. Fusion, 2015, 23: 99-115.

[3] Stathaki T. Image Fusion: Algorithms and Applications[M]. New York: Academic Press, 2008.

[4] KONG W W, LEI Y, ZHAO H X. Adaptive fusion method of visible light and infrared images based on non-subsampled shearlet transform and fast non-negative matrix factorization[J]. Infrared Phys. Techn., 2014, 67: 161-172.

[5] Fernando Mendozaa, LU Renfu, CEN Haiyan. Comparison and fusion of four nondestructive sensors for predicting apple fruit firmness and soluble solids content[J]. Postharvest Biol. Technol., 2012, 73: 89-98.

[6] Eslami M, Mohammadzadeh A. Developing a spectral-based strategy for urban object detection from airborne hyperspectral TIR and visible data[J]. IEEE Journal of Selected Topics in Applied Earth Observations \& Remote Sensing, 2016, 9(5): 1808-1816.

[7] HAN Lei, Buzha Wulie, YANG Yiling, et al. Direct fusion of geostationary meteorological satellite visible and infrared images based on thermal physical properties[J]. Sensors, 2015, 15(1): 703-714.

[8] SCHMIDT R. Benefits of IR/visible fusion[C]//Proceedings of SPIE, 2007, 6541: 654105-1-654105-6.

[9] Gemma Piella. A general framework for multiresolution image fusion: from pixels to regions[J]. Inform. Fusion, 2003(4): 259-280.

[10] ZHOU Z Q, LI S, WANG B. Multi-scale weighted gradient-based fusion for multi-focus images[J]. Inf. Fusion, 2014, 20(1): 60-72 .

[11] YU X, REN J, CHEN Q. A false color image fusion method based on multi-resolution color transfer in normalization YCBCR space[J]. Optik-International Journal for Light and Electron Optics, 2014, 125(20): 6010-6016..

[12] LIU Y, LIU S, WANG Z. A general framework for image fusion based on multi-scale transform and sparse representation[J]. Information Fusion, 2015, 24: 147-164.

[13] 李婵飞, 邓奕. 平稳小波变换和模糊数学的红外与可见光图像融合 [J]. 计算机与数字工程, 2017, 45(5): 39-42.
LI Chanfei, DENG Yi. Infrared and visible light image fusion based on stationary wavelet transform and fuzzy mathematics[J]. Computer and Digital Engineering, 2017, 45 (5): 39-42.

[14] LI He, LIU Lei, HUANG Wei, et al. An improved fusion algorithm for infrared and visible images based on multi-scale transform[J]. Infrared Phys. Technol., 2016, 74: 28-37.

[15] LI Huafeng, QIU Hongmei, YU Zhengtao, et al. Infrared and visible image fusion scheme based on NSCT and low-level visual features[J]. Infrared Phys. Technol., 2016, 76: 174-184.

[16] HAN Liang, LI Chanfei, PU Xiujuan. Image fusion method for infrared and visible images utilizing entropy of the histogram and stationary wavelet transform[J]. Journal of Computational Information Systems, 2013, 9(10): 3883-3892.

[17] 韩亮, 李婵飞, 蒲秀娟. 图像分割与平稳小波变换法融合红外与可见 光图像[J]. 重庆大学学报, 2013, 36(6): 112-118.

HAN Liang, LI Chanfei, PU Xiujuan. Image fusion method for infrared and visible light images based on image segmentation and stationary wavelet transform[J]. Journal of Chongqing University, 2013, 36(6): 112-118.

[18] Vapnik V. An overview of statistical learning theory[J]. IEEE Transaction on Neural Networks, 1999, 10(5): 988-999.

[19] 崔景泰. 小波导论[M]. 西安: 西安交通大学出版社, 1995. CUI Jingtai. Introduction to Wavelet[M]. Xi'an: Xi'an Jiaotong University Press, 1995.

[20] 杨丹, 何建农. 基于 NSCT 变换的红外与可见光图像融合新算法[J]. 福州大学学报: 自然科学版, 2017, 45(5): 635-640.

YANG Dan, HE Jiannong. New algorithm for infrared and visible light image fusion based on NSCT transformation[J]. Journal of Fuzhou University: Natural Science Edition, 2017, 45 (5): 635-640.

[21] YAN X, QIN H, LI J, et al. Infrared and visible image fusion with spectral graph wavelet transform[J]. Journal of the Optical Society of America A Optics Image Science \& Vision, 2015, 32(9): 1643-52.

[22] JIN X, JIANG Q, YAO S, et al. A survey of infrared and visual image fusion methods[J]. Infrared Physics \& Technology, 2017, 85: 478-501. 